\title{
The Marshall Space Flight Center Development of Mirror Modules for the ART-XC Instrument aboard the Spectrum-Roentgen-Gamma Mission
}

\author{
M. Gubarev ${ }^{1}$, B. Ramsey ${ }^{1}$, S.L. O’Dell ${ }^{1}$, R. Elsner ${ }^{1}$, K. Kilaru ${ }^{1}$, J. McCracken ${ }^{1}$, \\ M. Pavlinsky ${ }^{2}$, A. Tkachenko², I. Lapshov² \\ ${ }^{1}$ NASA Marshall Space Flight Ctr., Huntsville, AL 35812, USA \\ ${ }^{2}$ Space Research Institute, Moscow, Russia
}

\begin{abstract}
The Marshall Space Flight Center (MSFC) is developing X-ray mirror modules for the ART-XC instrument on board the Spectrum-Roentgen Gamma Mission under a Reimbursable Agreement between NASA and the Russian Space Research Institute (IKI.) ART-XC will consist of seven co-aligned X-ray mirror modules with seven corresponding CdTe focal plane detectors. Currently, four of the modules are being fabricated by the Marshall Space Flight Center (MSFC.) Each MSFC module consist of 28 nested Ni/Co thin shells giving an effective area of $65 \mathrm{~cm}^{2}$ at $8 \mathrm{keV}$, response out to $30 \mathrm{keV}$, and an angular resolution of 45 arcsec or better HPD. Delivery of these modules to the IKI is scheduled for summer 2013. We present a status of the ART x-ray modules development at the MSFC.
\end{abstract}

\section{SRG Overview}

The Spectrum-Röntgen-Gamma (SRG) mission is a Russian-German X-ray astrophysical observatory that carries two co-aligned and complementary X-ray telescope systems. The primary instrument is the German-led extended ROentgen Survey with an Imaging Telescope Array (eROSITA) ${ }^{1}$, a 7-module X-ray telescope system that covers the energy range from 0.2-12 keV. The complementary instrument is the Russian-led Astronomical Roentgen Telescope $-\mathbf{X}$-ray Concentrator (ART-XC or ART) ${ }^{2}$, a 7-module X-ray telescope system that provides higher energy coverage, up to 30 $\mathrm{keV}$ (with limited sensitivity above $12 \mathrm{keV}$ ). Table 1 and Figure 1 compare key performance characteristics of the eROSITA and ART telescope systems. SRG's primary mission is to perform the most sensitive X-ray all-sky survey (4 years) to be followed with pointed observations ( 3 years).

The SRG observatory will be launched into an approximately low-Earth orbit from Baikonur and then delivered to a 6month-period halo orbit around the outer Lagrange point (L2) via a Zenit rocket and Fregat booster. To optimize the final orbital parameters, a gravitational maneuver around the Moon will be performed.

Table 1. Performance characteristics of the ART and eROSITA instruments aboard the SRG mission.

\begin{tabular}{|c|c|c|}
\hline Parameter & ART & eROSITA \\
\hline $\begin{array}{l}\text { Energy Range } \\
\text { Effective Area } \\
\text { Field of View } \\
\text { System Angular Resolution (on axis) } \\
\text { Energy Resolution }\end{array}$ & $\begin{array}{c}5-30 \mathrm{keV} \\
455 \mathrm{~cm}^{2} \text { at } 8 \mathrm{keV} \\
32 \operatorname{arcmin} \\
1 \text { arcmin } \\
1.4 \mathrm{keV} \text { at } 14 \mathrm{keV}\end{array}$ & $\begin{array}{c}0.2-12 \mathrm{keV} \\
2500 \mathrm{~cm}^{2} \text { at } 1 \mathrm{keV} \\
1 \mathrm{deg} \\
15 \text { arcsec } \\
130 \mathrm{eV} \text { at } 6 \mathrm{keV}\end{array}$ \\
\hline
\end{tabular}

*Mikhail.V.Gubarev@nasa.gov; Phone: 1256 544-7816; FAX 1256 544-2659 

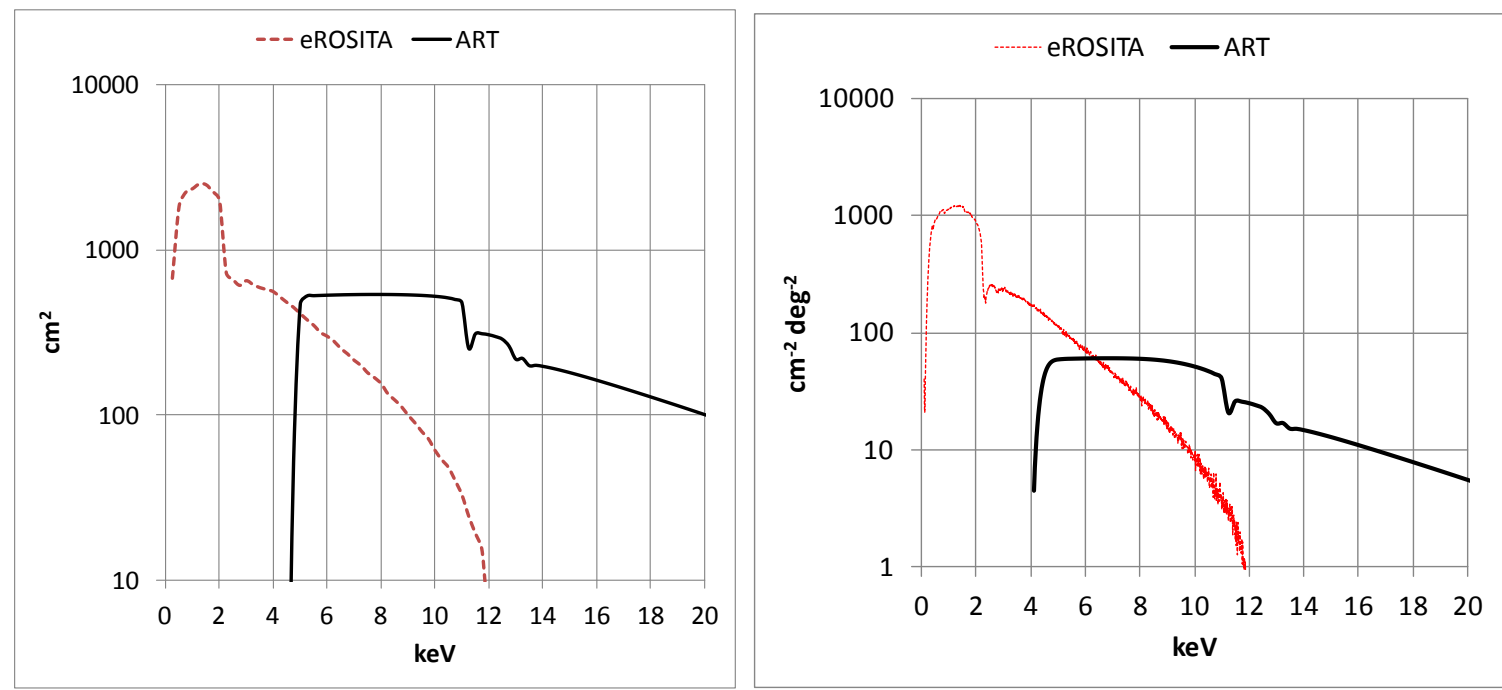

Figure 1. (Left) A comparison of the net (optics with detector) on-axis effective area $A_{\text {eff }}(E, 0)$ of the eROSITA and the ART telescope systems aboard SRG. (Right) A comparison of the net (optics with detector) effective grasp $\mathrm{G}_{\text {eff }}(\mathrm{E})$ of the eROSITA and the ART telescope systems aboard SRG.

The SRG observing program is divided into three stages over the 7.5-year mission lifetime. The first $\sim 100$ days, during the transit to the L2 point, will be used for initial check-out and in-flight calibrations. The next 4 years will be devoted to an all-sky survey, during which time the spacecraft rotates about an axis that points, initially, directly towards the sun. Thus, the overlapping point of the great scan circles gives a deep survey region at each ecliptic pole. An additional component, a slight change of the rotational axis by 8 deg every 6 months, results in a total of 8 deep field survey regions in each hemisphere. One region, described above, coincides with the Ecliptic pole and 7 additional fields are symmetrically positioned on an 8 degree radius circle from the pole, so the solid-angle of each deep-survey region is broadened to approximately $200 \mathrm{deg}^{2}$ (for details of the exposure map for the 4 year survey see Pavlinsky, et al. "The ART-XC Instrument on board the SRG Mission" in the proceedings of this conference.) SRG will observe the whole sky every half year due to Earth's revolution around the Sun. The final stage of the mission, which will last about 3 years, will be spent on pointed observations.

Table 2. ART flight mirror module parameters

\begin{tabular}{|c|c|}
\hline Parameter & Value \\
\hline Number of Mirror Modules & $7=4+3$ \\
\hline Number of Shells per & 28 \\
\hline Module & \\
\hline Shell Coating & $\begin{array}{l}>10 \mathrm{~nm} \text { of iridium }(>90 \% \\
\text { bulk density) }\end{array}$ \\
\hline Shell Total Length & $580 \mathrm{~mm}$ \\
\hline $\begin{array}{l}\text { Encircled Half Energy } \\
\text { Width }\end{array}$ & $\begin{array}{l}\text { Less than } 1 \mathrm{~mm} \text { diameter, } \\
\text { center of field of view }\end{array}$ \\
\hline & $\begin{array}{l}\text { Less than } 2.5 \mathrm{~mm} \text { diameter, } \\
15 \text { arcmin off axis }\end{array}$ \\
\hline $\begin{array}{l}\text { Mirror Module Effective } \\
\text { Area }\end{array}$ & $\geq 65 \mathrm{~cm}^{2}$ at $8 \mathrm{keV}$ (on axis) \\
\hline Module Focal Length & $\begin{array}{l}2700 \pm 1 \mathrm{~mm} \text { from mirror } \\
\text { intersection plane }\end{array}$ \\
\hline $\begin{array}{l}\text { Allowable Total Mass per } \\
\text { Module }\end{array}$ & $\begin{array}{l}17 \mathrm{~kg} \text { including thermal } \\
\text { control system }\end{array}$ \\
\hline $\begin{array}{l}\text { Operating Temperature } \\
\text { Range }\end{array}$ & $17^{0} \mathrm{C}$ to $23^{\circ} \mathrm{C}$ \\
\hline
\end{tabular}

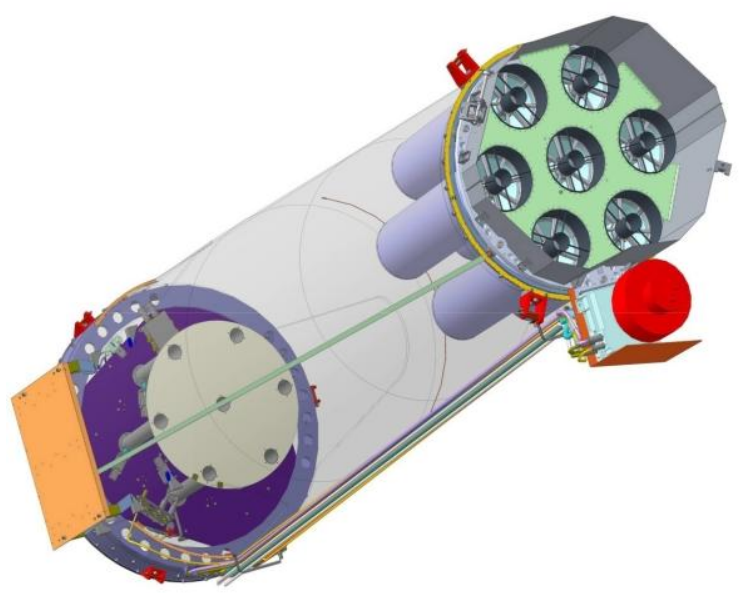

Figure 2. The ART Instrument with seven mirror modules and seven focal-plane detectors. 


\section{ART-XC Instrument}

The ART instrument comprises seven independent, co-aligned telescopes (mirror assemblies and detectors) sharing a carbon-fiber optical bench, which is equipped with a moveable cover to protect the optics during launch (see Figure 2). An active thermal-control system maintains the mirror modules in their desired near-room-temperature operating range,

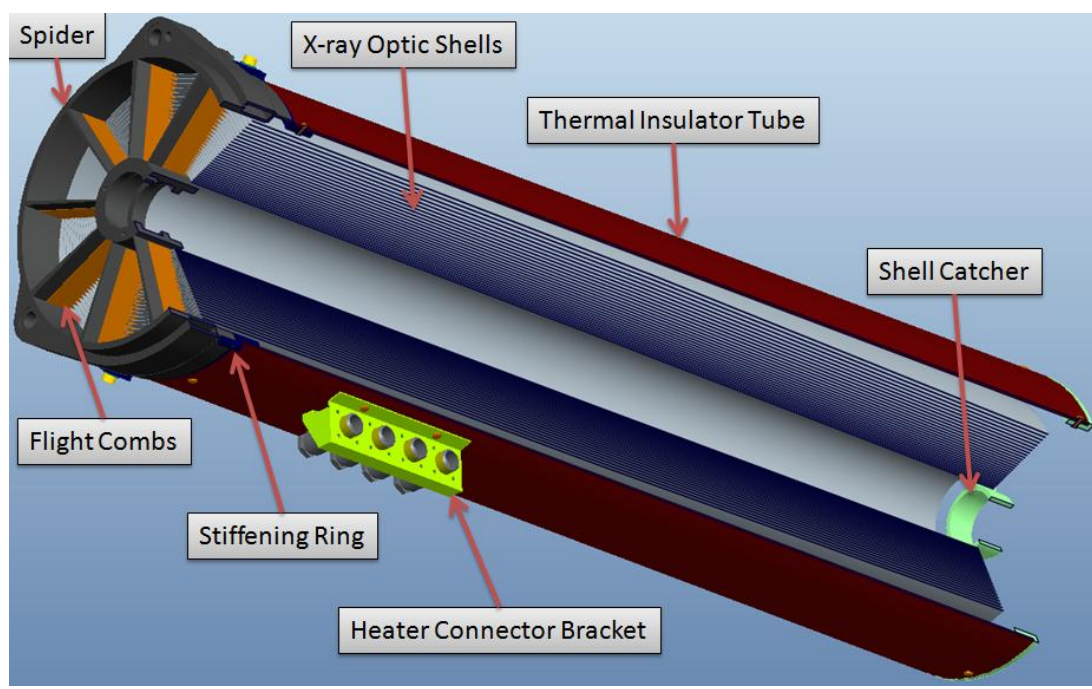

Figure 3. A cross section of an ART X-ray mirror module. The inner baffle and the heaters are not shown whereas a passive system (the radiator for which is shown at the bottom left of Figure 2) keeps the focal-plane detectors at their required temperature.

MSFC has designed and is fabricating four ART x-ray optics modules under an International Reimbursable Agreement between NASA and with IKI.

Figure 3 displays a sectional schematic of an ART mirror-module assembly designed by MSFC. Each module comprises 28 concentrically nested shells, fabricated from a nickel-cobalt alloy. The shells vary in thickness from $250 \mu \mathrm{m}$ (inner) to $350 \mu \mathrm{m}$ (outer) and range in diameter from about $50 \mathrm{~mm}$ to $150 \mathrm{~mm}$. The total length (primary and secondary mirror surfaces combined) is $580 \mathrm{~mm}$. A thin coating (> $100 \mathrm{~nm}$ ) of near-bulk-density iridium sputtered onto the inner surfaces enhances the high-energy reflectivity.

For each mirror module, a single spider holds the mirror shells at one end only through combs glued onto the spider legs. A thin aluminum thermal insulator tube with heaters maintains the module within the desired $\pm 3^{\circ} \mathrm{C}$ operational temperature range. Three attachment lugs on the periphery of the spider secure each module to the support plate on the top of the optical bench.

Figure 3 shows the expected effective area of a single mirror module (no detector response or thermal insulation) for various off-axis angles. The science-derived effective area requirement for the optics is $>65 \mathrm{~cm}^{2}$ at $8 \mathrm{keV}$ on axis, and thus the current design exceeds that value. Table 2 lists other requirements, generated by IKI, for the mirror module. The required angular resolution is approximately 1 arcminute (half energy width) and readily satisfied: MSFC-produced Xray mirror modules are typically at least a factor of two better than this, so the goal angular resolution for the ART mirror module is 30 arcseconds.

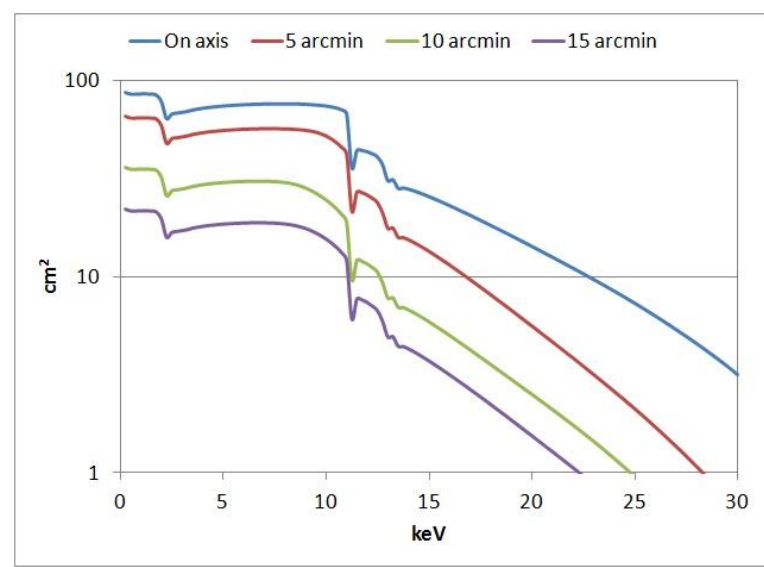

Figure 3. ART single mirror module effective area vs energy for various off-axis angles.

\section{MSFC Infrastructure}

MSFC is utilizing electroformed-nickel replication (ENR) to fabricate four ART X-ray mirror modules ${ }^{3,4}$. In this process a thin nickel or nickel-alloy mirror shell is electroformed onto a figured and super-polished electroless-nickelplated aluminum mandrel, from which it is subsequently separated in chilled water by differential thermal contraction. One attraction of this ENR process is that the resulting full-shell mirrors are inherently rigid, fostering good angular resolution. 


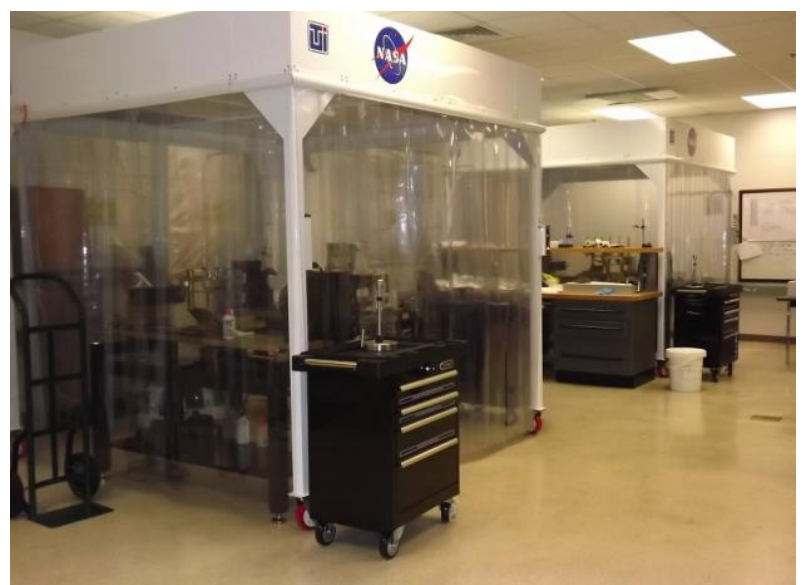

Figure 4. ART polishing stations under clean room tents in the mandrel production area.

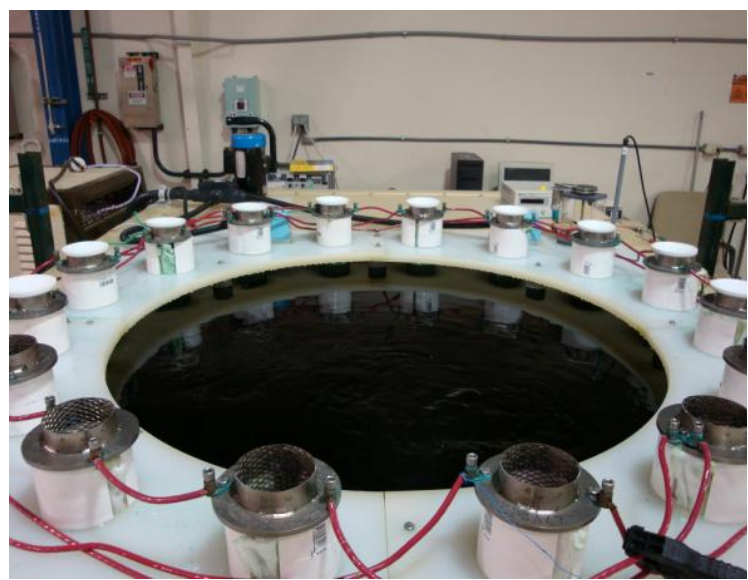

Figure 5. One of the two electroformed-nickel-replication baths dedicated to the ART XC program.

Production of a flight X-ray mirror module entails five tasks: (1) mandrel production, (2) shell electroforming, (3) reflective (iridium) coating, (4) mirror-module assembly, and (5) module calibration and testing. For ART, MSFC expanded its capability to accommodate the increased workload and aggressive production schedule. The most laborintensive and, hence, most expensive process is the mandrel fabrication. MSFC has assembled five polishing stations to ensure completion of the 28 ART mandrels on schedule. Figure 4 shows the mandrel polishing area, with polishing stations installed under clean-room tents.

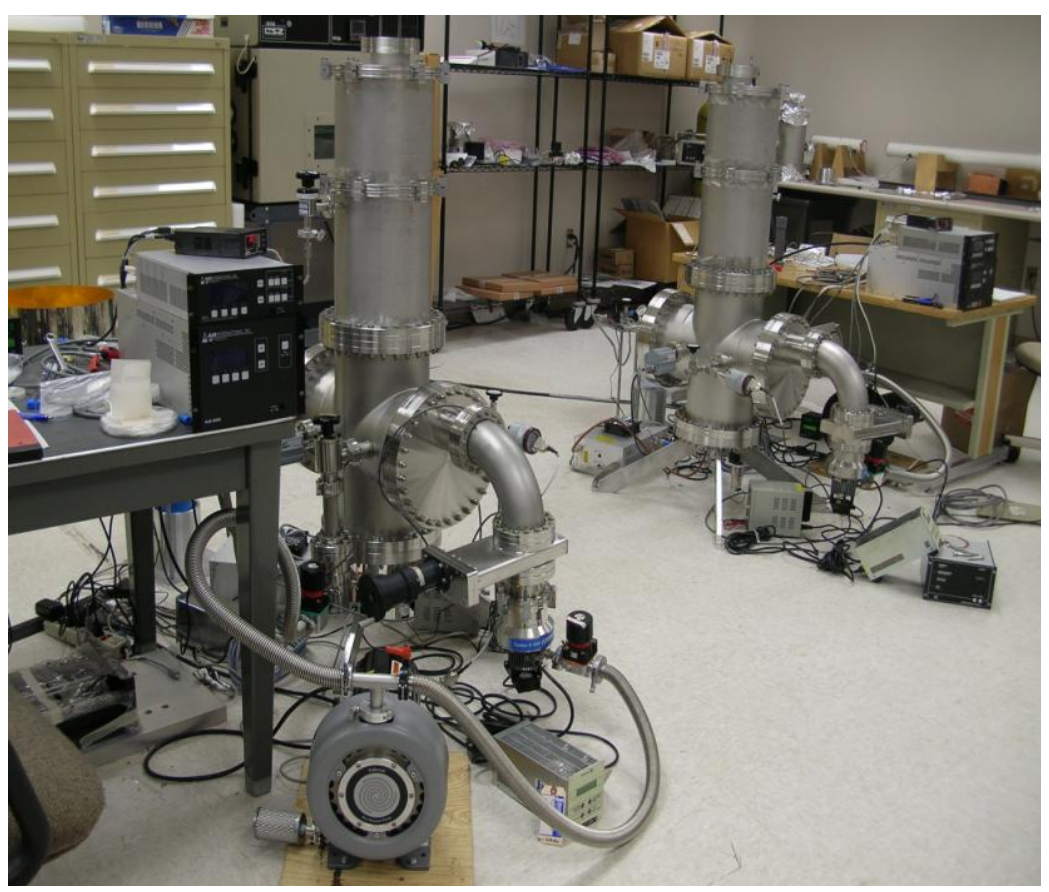

Figure 6. Two ART iridium coating chambers being characterized.
To meet the ART required mirrorshell-production rate, MSFC constructed an additional electroforming bath (Figure 5), giving a total capacity of 810 shells per week. To keep pace with this production, we developed two custom radio-frequency (RF) sputtering chambers to coat a thin $(10 \mathrm{~nm})$ layer of iridium inside shells (see Figure 6), prior to mirror-module assembly.

Once the shells are replicated and coated, they must be assembled into mirror modules. To enable multiple modules to be assembled as shells become available, we are fabricating five alignment and assembly stations. The stations are scaled down versions of the alignment system (Figure 7) developed at MSFC for the Berkeley-led FOXSI (Focusing Optics X-ray Solar Imager) ${ }^{5}$ rocket program. Upon completion of the new stations, we expect to be able to mount and glue 1 shell per day on each of the five systems. 

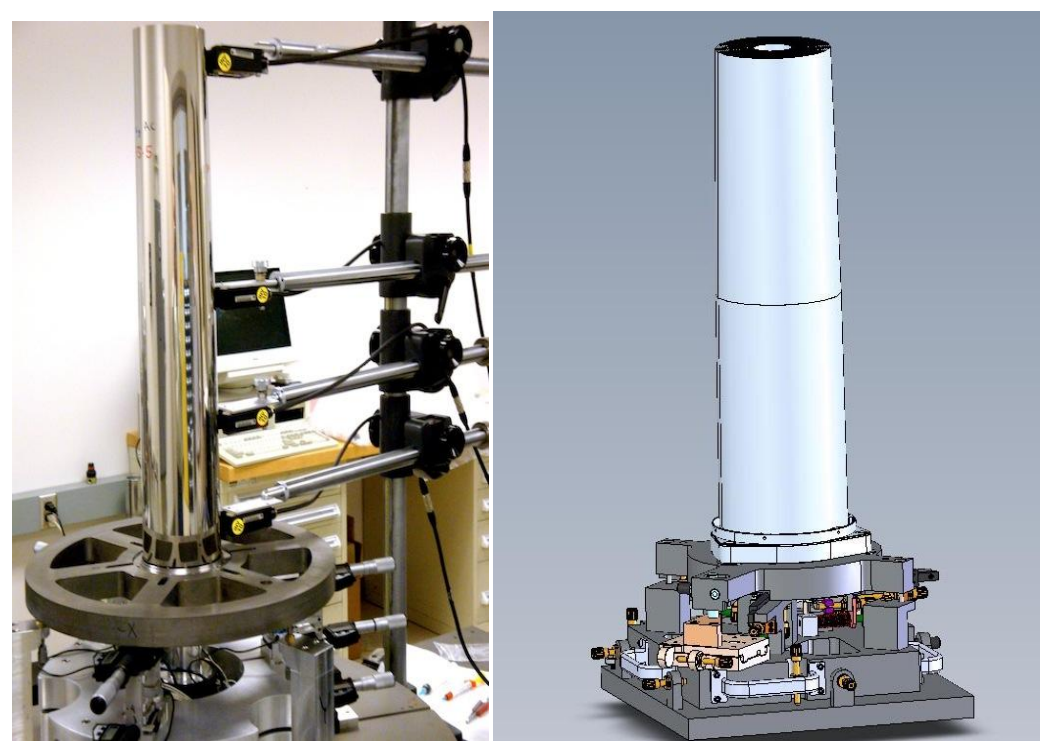

Figure 7. The x-ray optics module alignment station used for the FOXSI project (left). This station was used as the prototype for the ART alignment station design shown on right. Five ART stations are being built.

After assembly of the flight modules, MSFC will vibration test them in its environmental test facility, to acceptance levels provided by IKI. Upon completion of vibration testing, each mirror module will be X-ray tested and calibrated in MSFC's 104-m-long MSFC Stray Light Facility, prior to shipment to Russia.

\section{Fabrication Status}

The housing of the ART modules has been designed to fulfill optical performance and mechanical stability requirements. The ART qualification unit which imitates the flight module resonance frequencies but with only six mirror shells and mass-simulators, has been designed and is being fabricated. After completion this engineering unit will be checked via xray measurements, environmentally tested (vibration, shock, acoustic and thermal), and then fully characterized in $\mathrm{x}$ rays prior to shipment to IKI.

The aluminum blanks for all 28 ART mandrels have been fabricated and coated with electroless nickel alloy. The quality of the coating and the mandrel dimensions have been verified at the MSFC. Thirteen mandrels have been figured using the diamond turning process and ten of these are in polishing. The six mandrels needed for the qualification unit are at the superpolishing stage.

Both electroforming baths are running and are currently being characterized. The iridium coating chambers have been calibrated and will be installed in close proximity to the electroforming baths, so that shells can be coated as soon as they become available. The new ART alignment stations are being fabricated. A modified FOXSI alignment station will be used for an early pre-qualification test unit while the ART stations are completed. After the ART stations are assembled and tested the FOXSI alignment station will be used as a backup.

MSFC has obtained an export license from the US State Department for shipment of the qualification and four flight units, as well as the supporting data to Russia. 


\section{Conclusions}

MSFC is developing four $\mathrm{x}$-ray mirror modules for the ART-XC instrument on board the SRG Mission under an International Reimbursable Agreement between NASA and the IKI. Delivery of these modules to IKI is scheduled for summer 2013 for a scheduled launch in 2014.

To support the aggressive ART module fabrication schedule, MSFC has greatly expanded its infrastructure to meet the demands of mandrel polishing, shell fabrication, shell coating, and module assembly. MSFC is on schedule to deliver flight units in the Summer of 2013.

\section{6. $\quad$ References}

[1] Predehl P., et al., "eROSITA on SRG", Proc. SPIE 7732, 77320U (2010).

[2] Pavlinsky M., et al. "The ART-XC Instrument on board the SRG Mission", Proc. SPIE 8147, 814706-814706-6 (2011).

[3] BD Ramsey, RF Elsner, D Engelhaupt, MV Gubarev, JJ Kolodziejczak, SL ODell, et al. The development of hard Xray optics at MSFC, Proc. of SPIE. 4851, 631-638 (2003).

[4] BD Ramsey, “Replicated Nickel Optics for the Hard-X-Ray Region”, Exp. Astron. 20, 85-92 (2005)

[5] Christe, S. et al. The Focusing Optics X-ray Solar Imager (FOXSI), Proc. SPIE, 8147, 814705 (2011). 